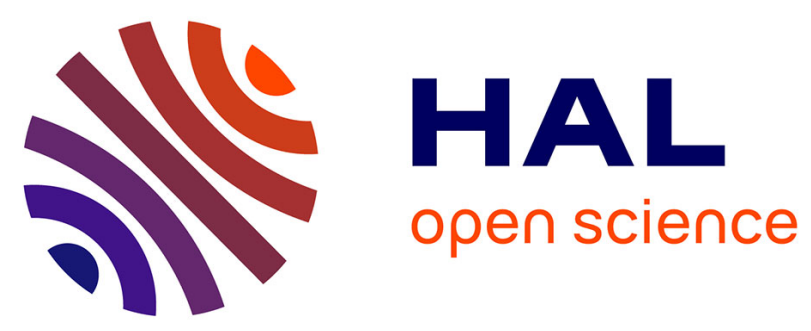

\title{
OBTAINING ELECTRON ENERGY LOSS SPECTRA AND X-RAY EMISSION SPECTRA FREE OF INSTRUMENTAL ARTIFACTS
}

\author{
A. Craven, P. Adam, W. Nicholson, J. Chapman, R. Ferrier
}

\section{- To cite this version:}

A. Craven, P. Adam, W. Nicholson, J. Chapman, R. Ferrier. OBTAINING ELECTRON ENERGY LOSS SPECTRA AND X-RAY EMISSION SPECTRA FREE OF INSTRUMENTAL ARTIFACTS. Journal de Physique Colloques, 1984, 45 (C2), pp.C2-437-C2-440. 10.1051/jphyscol:1984299 . jpa00224014

\section{HAL Id: jpa-00224014 https://hal.science/jpa-00224014}

Submitted on 1 Jan 1984

HAL is a multi-disciplinary open access archive for the deposit and dissemination of scientific research documents, whether they are published or not. The documents may come from teaching and research institutions in France or abroad, or from public or private research centers.
L'archive ouverte pluridisciplinaire HAL, est destinée au dépôt et à la diffusion de documents scientifiques de niveau recherche, publiés ou non, émanant des établissements d'enseignement et de recherche français ou étrangers, des laboratoires publics ou privés. 


\title{
OBTAINING ELECTRON ENERGY LOSS SPECTRA AND X-RAY EMISSION SPECTRA FREE OF INSTRUMENTAL ARTIFACTS
}

\author{
A.J. Craven, P.F. Adam, W.A.P. Nicholson, J.N. Chapman and R.P. Ferrier \\ Department of Natural Philosophy, University of GLasgow, GLasgow G12 8QQ, \\ scotzand
}

Résumé - L'enregistrement des spectres EDX ou EELS est entaché d'erreurs dues àl'instrument. Si possible, celles-ci doivent être éliminées; sinon, les spectres doivent être corrigés.

Abstract - When recording EDX and EELS spectra instrumental artifacts occur. If possible, they should be eliminated, if not the spectra should be corrected.

\begin{abstract}
When analysing small volumes of material using a fine electxon probe on thin specimens, it is of great benefit to record the available signals simultaneously especially if radiation damage, specimen drift or contamination are the limiting factors. Electron energy loss spectroscopy (EELS) and energy dispersive $x-r a y$ spectroscopy (EDX) make use of two such signals and between them, they provide information over a wide range of atomic number. In recoraing the data, it is essential to minimise the instrumental artifacts and be able to correct the residual effects. This and subsequent processing of the data are most easily carried out if the data are stored in digital form. We have used a digital acquisition system from Toltec Computer for simultaneous EELS and EDX in our VG Microscopes HB5 scanning transmission electron microscope which is fitted with a Link systems $S_{i}$ (Ii) detector and nucleonics. We have built the necessary hardware interfaces to provide a digital scan for the electron spectrometer and convert the output from the spectrometer to a digital form suitable for the computer $/ 1 /$. It is also a powerful image acquisition system $/ 2 /$.
\end{abstract}

For both EELS and EDX, the incident probe convergence should be defined well away from the specimen and the spurious radiation intercepted by a spray aperture. The HB5 achieves this very efficiently by using an aperture immediately after the gun to define the beam and a very small aperture in the plane of an intermediate crossover as the spray aperture. Thus the x-ray "hole count" is negligible, as is the stray background in the EELS spectrum from electrons outside the incident probe.

In the case of EELS, the scattering distribution leaving the specimen is matched to the acceptance angle of the spectrometer using post-specimen lenses /3/. For a thin specimen the current in the zero loss peak can approach that in the incident probe, typically $\sim \mathrm{I} \mathrm{n} \cdot \mathrm{A}$ in the HB5. Thus even the fastest pulse counting system will not work and the analogue signal must be digitised. However, at large energy losses the signal may drop below $10^{-15}$ where photomultiplier dark current is a problem. At this level pulse counting is most suitable and the dark current can be eliminated using a discriminator. As the signal level increases, the detection efficiency falls because of the deadtime from the finite pulse width. Thus neither technique is ideal over the whole range but both are approximately correct in the mid-range of signals. If both are available they can be used to correct each other. They can be obtained simultaneously by using output pulses of very low amplitude from the preamplifiex and feeding them into a low noise pulse amplifier/discriminator. The preamplifier output is also fed to a voltage to frequency converter which digitises the analogue signal producing a series of pulses. As the pulses used for the pulse amplifier have low amplitude, the preamplifier does not saturate until the signal level is 1000 times that required to cause severe pulse overlap. This increases 
the dynamic range by this factor. The pulses from each output are counted for the dwell period and then read by the computer. Fig 1 shows the signals recorded from a thin silicon film as a $\log \log$ plot. Saturation of the pulse signal is seen at high signal levels while the analogue signal tends to a noisy constant value at low signal levels. A region of agreement is seen in the mid-range, which is highly compressed by the logarithmic scale. By subtracting off the constant level from the analogue signal and plotting the ratio of the two signals against energy loss (Fig 2) the digitisation noise of the analogue signal can be seen on the right, a relatively constant region in the middle and a rapid falloff on the left. One could take the plateau level as the scaling factor and merge the two signals at one point but the analogue signal would then be relatively low at the merge point and the digitisation exror quite important. A better approach is to fit an ideal deadtime relationship over a region where the noise is not too large. Such a procedure works satisfactorily provided the ratio in the fitting region does not drop below 0.6 of the maximum value. An example of a fit can be seen in Fig 2 where the fitting region was from 145 to $300 \mathrm{eV}$ and the fit has been extrapolated to the end of the spectrum. The fit gives both the scaling factor and the deadtime. If the signal exceeds that at the start of the fit region (145 ev in Fig 2), the analogue signal can be scaled and used. If not, the pulse signal can be corrected for the deadtime and used. At the same time a correction can be made for the small amount of stray scattering in the spectrometer /4/. Fig 3 is the resulting spectrum corrected for the majority of the instrumental artifacts.

In the case of EDX, the considerations are somewhat different. When the electron probe hits the specimen, scattering over a wide angular range occurs. Electrons scattered through $290^{\circ}$ travel in approximately circular orbits in the strong axial magnetic field of the objective lens. For a modern high excitation lens the orbit diameter is $3-4 \mathrm{~mm}$. If these electrons strike solid material such as a specimen grid, the material of the cartridge or the thick edge of a specimen thinned from bulk material, they generate $x$-rays with a much greater efficiency than in the thin area of the specimen, thus giving an appreciable contribution to the spectrum. Even if these electrons do not hit solid material as they leave the specimen, they are very likely to be scattered back to the specimen region if they hit distant material at glancing angle. This might be a polepiece or anticontaminator whose distance from the axis is less than the cyclotron diameter of the electron orbit. Thus it is essential to minimise the amount of solid material in the specimen region. Fig 4 is a spectrum recorded from a 1500A thick silver specimen on a 75 mesh titanium grid using the HB5 and a Link Systems 860 EDX system. The specimen was tilted $20^{\circ}$ towards the detector and the spectrum is an average of several taken at different points on the specimen, all at least $30 \mu \mathrm{m}$ from the grid bars. A spectrum averaged from different points on the solid grid and scaled to the titanium peak in the silver spectrum is also shown in Fig 4. Examination of this suggests that the contribution to the bremsstrahlung in the silver spectrum from stray scattering onto the solid grid bars is $25 \%$ and can be removed by subtraction. The cartridge used was constructed so that most of the solid material was outside both the cyclotron diameter of the electrons and the field of view of the collimator and thus it contributes negligible bremsstrahlung and only very small characteristic peaks to the spectrum. Use of a beryllium grid removes the characteristic titanium peak but leaves a similar contribution to the bremsstrahlung because of the increase in thickness and area of the grid bars. A spectrum using a beryllium grid is shown in Fig 5 . A theoretical bremsstrahlung shape, calculated using a modified Bethe-Heitler (MBH) equation, has been scaled to the region between 10 and $15 \mathrm{kev}$. /5/. Corrections were made for the finite detector resolution, absorption in the specimen and that in the detector window. The window thickness used was that given by the manufacturer, the film thickness was that measured during its preparation and the specimen tilt was that of the cartridge; these were not used as free parameters in the fit. The fit is good except on the low energy side of the silver I peaks where detector artifacts cause severe distortion of the specimen. If the beryllium double tilt cartridge supplied by VG Microscopes is used the contribution goes up a further 10 - 15\%. For most purposes this is not a problem but it is an indication of the magnitude of the contribution that arises from solid material. In other circumstances very appreciable characteristic peaks might result, for example when using specimens thinned from bulk material. 
Turning to the detector artifacts, the review article by statham $/ 6 /$ gives a good description of them. The poor fit of the theoretical background at low energy is mainly the result of incomplete charge collection by the detector causing counts from some silver I $x$-rays to appear over a wide range of lower energies. By subtracting an appropriately scaled spectrum obtained by fluorescing silver, the majority of this effect can be removed as shown in Fig 6 . The peak/dip at the silver $I$ energies is caused by a shift of about $10 \mathrm{eV}$ between the spectra. The MBH fit is now much better but the background itself has also been distorted by the same effect and needs to be corrected before the accuracy of the fit can be determined. Thus we can obtain spectra essentially free of microscope artifacts in the case of thin film specimens but detector artifacts still remain.

Idealiy the corrected spectra from both techniques ought to give identical results if they can detect the same elements. As a check on this, we have compared transition metal concentration ratios obtained by analysing carbides extracted from steels using EDX and EELS. The MBH equation provides a good background stripping procedure for EDX but separating the contributions from closely spaced edges remains a problem in EELS. The preliminary results indicate a systematic error of $\sim 15 \%$ between the ratios obtained by EELS and EDX with larger errors when one element is present in low concentration $/ 7 /$.

1. CRAVEN A J, MACLEOD A M \& FERRIER R P. Electron Microscopy 1980, Proc 7th European Cong on E. M. eds. P Brederoo \& G Boom vol I 322

2. CHAPMAN $J N$ \& MORRISON $G$ R. PYOC EMAG 83 in press

3. CRAVEN A J, \& BUGGY T W. Ultramicroscopy 7 (1981) 27

4. CRAVEN A J, BUGGY $T$ W \& FERRIER R P. Quantitative Microanalysis with High Spatial Resolution (Book 227, Metals Society, London 1981) 141

5. CHAPMAN J N, GRAY C G, ROBERTSON B W \& NICHOLSON W A $P_{0}$ X-ray Spectrometry in press

6. STATHAM P J. J Microscopy 123 (1981) 1

7. CROZIER P A, CHAPMAN J N, CRAVEN A J \& TITCHMARSH J M. PrOC EMAG 83 in press
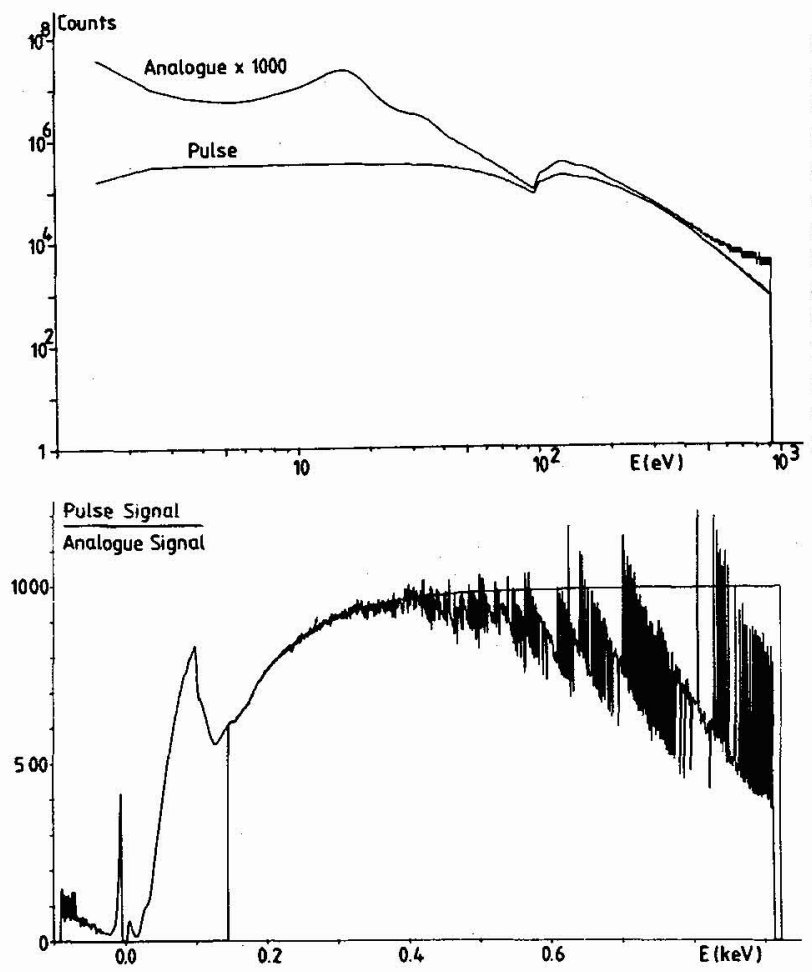

Fig 1 Simultaneous pulse and analogue EELS spectra from a thin silicon film. The pulse spectrum uses a low noise pulse amplifier/discriminator to count individual electrons. The analogue spectrum uses a voltage to frequency converter to digitise the output current of the photomultiplier. A logarithmic scale is used to show the dynamic range. The intensity in the zero loss peak is $1.8 \times 10^{8}$ counts.

Fig 2 Ratio of the pulse spectrum to the analogue spectrum, which has been corrected for a constant dark current. A fit has been made in the region from 145 to $300 \mathrm{eV}$ assuming an ideal deadtime relationship. This fit has been extrapolated to the end of the spectrum. 

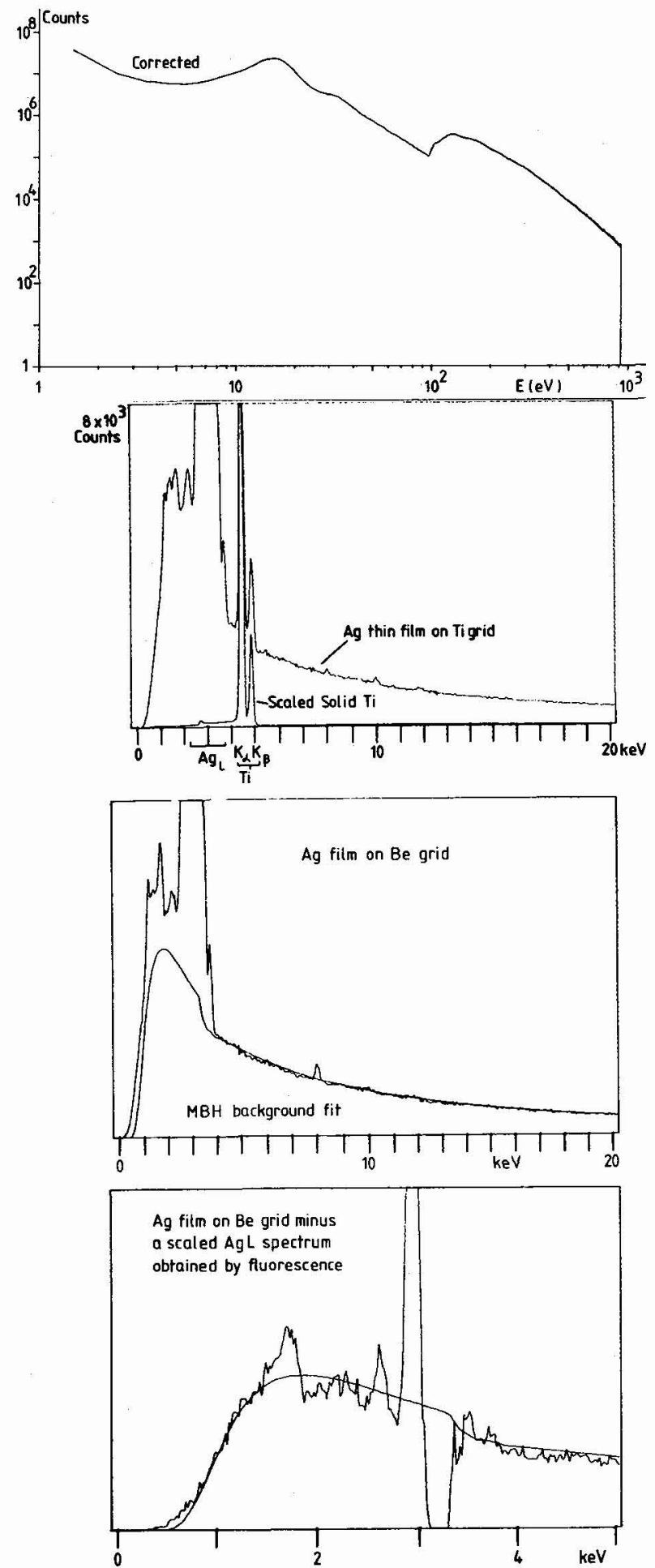

Fig 3 The corrected spectrum obtained by using the scaled analogue signal when the input signal is above that at $145 \mathrm{eV}$ and the pulse signal corrected for deadtime when the input signal is below this level.

Fig 4 X-ray spectrum from 1500 \& of silver backed by $500 \AA$ of formvar supported on a 75 mesh titanium grid. The small solid support arms in the cartridge were aluminium. A spectrum from the solid grid bar, scaled to the titanium peak in the thin film spectrum, is also shown.

Fig 5 X-xay spectrum from a similar film on a 75 mesh beryllium grid. The small solid support arms in the cartridge were copper. A theoretical background fit calculated using a modified Bethe-Heitler formula is also shown. The effect of incomplete charge collection in the detector can be seen on the low energy side of the silver I peaks.

Fig 6 The spectrum in fig. 5 minus a spectrum obtained by fluorescing silver scaled to the silver I peak. The majority of the incomplete charge collection and the silicon escape peaks from the silver $\mathrm{L}$ peaks are thus removed giving much closer agreement between experiment and theory except in the region of the silicon absorption edge. (The peak and dip near the position of the silver I peaks are caused by w10 eV shift between the two spectra.) 